\title{
Pain Assessment among African Neonates
}

\author{
Desire Aime Nshimirimana ${ }^{1,}$, , Donald Kokonya ${ }^{2}$, Jeanne Marie Claude Uwurukundo ${ }^{3}$, \\ Phocas Biraboneye ${ }^{4}$, Fred Were ${ }^{5}$, Cyprien Baribwira ${ }^{3}$ \\ ${ }^{1}$ Department of Health Systems Management, School of Medicine \& Health Sciences, Kenya Methodist University, Nairobi, Kenya \\ ${ }^{2}$ Department of Mental Health, School of Medicine, Masinde Muliro University of Science \& Technology, Kakamega, Kenya \\ ${ }^{3}$ Department of Pediatrics \& Neonatology, School of Medicine, National University of Rwanda, Butare, Rwanda \\ ${ }^{4}$ Department of Obstetrics and Gynecology, School of Medicine, University of Nairobi, Nairobi, Kenya \\ ${ }^{5}$ Department of Paediatrics \& Child Health, School of Medicine, College of Health Sciences, University of Nairobi, Nairobi, Kenya
}

\section{Email address:}

nshima2007@yahoo.fr (D. A. Nshimirimana),dkokonya@yahoo.com (D. Kokonya), uwurukundo@gmail.com (J. M. C. Uwurukundo), biraboneye@gmail.com (P. Biraboneye), frednwere@gmail.com (F. Were), Cbaribwira.ihv@gmail.com (C. Baribwira)

*Corresponding author

\section{To cite this article:}

Desire Aime Nshimirimana, Donald Kokonya, Jeanne Marie Claude Uwurukundo, Phocas Biraboneye, Fred Were, Cyprien Baribwira. Pain Assessment among African Neonates. American Journal of Pediatrics. Vol. 2, No. 2, 2016, pp. 4-9. doi: 10.11648/j.ajp.20160202.11

Received: August 11, 2016; Accepted: August 29, 2016; Published: October 11, 2016

\begin{abstract}
Neonates who require treatment and venous drawing of blood samples in the newborn units are subjected to acute and painfully invasive procedures. Several tools to assess pain among newborns have been developed and are widely used in developed countries, but in Africa, there is limited experience in the assessment pain among newborns. This study assessed physiological and behavioral responses to pain among neonates during invasive procedures performed in a newborn unit in Rwanda. A total of 60 neonates born at gestational age of 28-42 weeks at the National University of Rwanda Teaching and Referral Hospital in the year 2005 were enrolled into this study. Blood pressures, heart and respiratory rates, oxygen saturation levels, the Neonatal Facing Coding System (NFCS) and Neonatal Acute Pain (APN) pain tools were and scores recorded before, immediately and 5, 10,15 and 20 minutes after procedures were recorded. Physiological parameters were compared using the Wilcoxon Signed Ranks Test while the NFCS and APN were compared using the McNemar Test. All (100\%) neonates experienced acutely peak pain in the first 5 minutes of the invasive procedures with peak responses recorded at 2.5 minutes and no pain (resolution) after 15 minutes among $81 \%$ of the neonates and only $6 \%$ experienced pain after 20 minutes. The increases in systolic blood pressures immediately after inflicting pain, 5, 10, 15 and 20 minutes were statistically significant $(\mathrm{p}<0.001, \mathrm{p}<0.001, \mathrm{p}<0.005$ and $\mathrm{p}<0.046)$ respectively compared to the diastolic blood pressures whose significant increases were at 5 and 10 minutes, $(p<0.001$ and $p<0.001)$ respectively. Respiratory rates were significantly high at the onset $(p<0.001), 5$ minutes $(p<0.001)$ and 10 minutes later $(p<0.002)$. Heart rates significantly increased at the onset of the procedures $(p<0.000), 5$ minutes $(p<0.001)$ and after 10 minutes $(p<0.033)$. Decrease in oxygen saturation immediately after the procedures was significant $(\mathrm{p}<0.001)$. Oxygen saturation immediately after the procedures significantly increased up to 5 minutes $(\mathrm{p}<0.001)$ and 10 minutes $(\mathrm{p}<0.001)$. Invasive procedures caused acute pain among neonates in the African settings but to date, neonatal practice had not been given its due consideration with the aim of reducing pain among African neonates.
\end{abstract}

Keywords: Pain, Assessment, Neonates, Physiological, APN, NFCS, Africa

\section{Introduction}

Pain management among neonates has changed drastically in the last ten years [1]. Previously and unknown, it was assumed or not appreciated that neonates did not experience pain because of incomplete myelination of their sensory nerves, immaturity of pain receptors, cortex and reduced localization of pain [2]. Studies on pain and stress among neonates have increased awareness about the subject [3]. However, the nociceptive pathways that detect and transmit information about pain to the brain are completely functional from the twentieth $\left(20^{\text {th }}\right)$ week of gestation and by extension; even the most premature of newborns can experience pain 
[4]. Within the neonatal departments, neonates who require treatment and drawing of blood samples are subjected to repeated, invasive and painful procedures [5]. Pain is associated with physiological, biochemical, behavioral and psychological alterations that can be recorded and to some extent, quantified [6]. It is reported that there may be up to $20 \%$ increase in the measurable physiological parameters in response to neonatal pain, according to the National Health and Medical Research of Australia [7]. Responses to pain among the neonates have successfully been accurately and reliably measured and quantified physiologically [8]. The neonatal cardiovascular, respiratory, hormonal and metabolic systems have been used to measure changes in the neonatal experience of pain and have been shown to be similar to those observed among adults, although greater in magnitude and shorter in duration [9]. These measurements, alongside certain consistent behavioural responses, are sensitive indicators of pain among the neonates [10]. The incapacity of the neonates to proportionately express pain makes effective management of pain challenging [11]. Pain among neonates is often underestimated or ignored, hence undertreated and mismanaged [12]. In addition, early exposure to pain has been shown to affect the way neonates respond to pain later in their lives [13]. Of the behavioural changes, the facial expression of the neonates to pain was considered the most reliable and consistent indicator of the magnitude and intensity of pain they felt and it had the least inter-observer disagreement as well [14]. Although the biochemical changes were perhaps the most sensitive quantifiable parameters, the need to use invasive methods was a deterrent and major drawback in its application [15]. There was evidence that a combination of physiological and behavioural changes provided a better estimate of pain among infants [16]. In order to introduce objectivity in the assessment of pain among neonates, various pain scales were designed and validated based either on physiological variations, behavioural changes or a combination of both [17]. Although the physiological and behavioral responses were sensitive indicators of pain, they had low specificity; and could occur with apprehension, stress related to diseases and discomfort [18]. The responses could also be altered by the physiological state of the neonates immediately preceding the painful stimulus, such as the stage of wakefulness, the time since it last fed and restraint techniques used among others [6, 18]. Despite these pitfalls, assessments of behavioural and physiological responses remain the most readily available, reliable and feasible methods of assessing pain among neonates [19]. Many tools have been developed to measure pain among neonates and have been widely used in developed countries but there had been inadequacy of experience in assessment of pain among neonatologists in Africa [20]. Several pain assessment tools have been revised but they are mainly designed for research purposes with hardly any conducted within African settings [21]. Despite the awareness about pain among neonates, most neonatal departments among hospitals in Rwanda had not been using any pain assessment tool and no study had been conducted to date to assess pain among neonates as part of improvement in neonatal care. The objective of this study was to assess and demonstrate pain experienced by neonates by monitoring neonatal physiological and behavioural responses to pain during and after invasive procedures commonly and procedurally performed in the neonatal departments in Rwanda. The findings of this study will help African neonatologists and paediatric practitioners to appreciate, measure, evaluate and manage pain among neonates accordingly in addition to policy formulation for action on neonatal pain management.

\section{Methodology}

This was a descriptive and cross sectional quantitative study to determine and quantify neonatal pain caused by the three key invasive clinical procedures commonly and procedurally performed on neonates in newborn units namely intravenous injections, phlebotomy and intramuscular injections. This study was conducted at a newborn unit in the Clinical Department of Neonatology at the National University of Rwanda Teaching and Referral Hospital over a 4-month period between September and December 2005. This study setting was located in the Southern Province of Rwanda, approximately $150 \mathrm{~km}$ South of Kigali (Capital City of Rwanda). As a University teaching and referral hospital, this department received pre- and term neonates from all over the Southern region of Rwanda. It was one of the two neonatal centres of excellence in Rwanda, managed by the National University of Rwanda and a suitable and appropriate site for this study. The University Ethics and Research Committee (ERC) gave approval for this study before enrolment of the participants. The sample size was 60 neonates hospitalized in the newborn unit who were born at gestational ages of between 28 and 42 weeks. The study participants were exclusive of medical complications including congenital and non-congenital anomalies and they had not been on any painkillers 12 hours prior to enrolment into the study in accordance with each day's neonatal management plans. Neonates born at a gestation of less than 28 and above 40 weeks, those with medical complications and on painkillers less than 12 hours to recruitment were excluded from this study. The confidence interval was set at $95 \%$, the margin error at $5 \%$ and the prevalence was taken to be $50 \%$ since no other study had been conducted on the same population. The sample size was calculated using the Cochran formula, 1977 [22]. The informed and voluntarily signed consent forms to participate in the study were granted in writing by the mothers of the neonates after providing adequate information about the study objective, procedures, protocols, harms and benefits to them. The behavioural aspect of pain was measured using a 10-item scale, the Neonatal Acute Pain (APN) [23] and an 8-item Scale, the Neonatal Facing Coding System (NFCS) [24]. The APN is a behavioural scale developed to rate acute pain in term and preterm neonates with a pain intensity scale range of $0-10$. It evaluated 10 items based on facial expressions, limb 
movements and vocal expressions. The intensity of pain was scored as no pain (0), moderate (1-3), severe (4-6) and very severe (7-10). Like the APN, the NFCS also measured the degrees of pain with the following parameters; brow bulge, squeezed eyes, nasolabial (smile or laugh lines) furrow, open lips, stretched mouth, lip purse, taut tongue, and chin quiver (tremble). This one scores pain as no pain (0), moderate (13 ), severe (4-6) and very severe (7-8). The APN scale evaluated pain with 10 points while the modified NFCS evaluates pain with 8 points. Pain increased proportionally to pain scores. Pain was graded from none, moderate, severe and very severe. These scales were used for both preterm and term neonates. In order to increase accuracy of assessment of pain, an automatic monitor was used to record physiological parameters namely blood pressures, heart rates, respiratory rates and oxygen saturation levels. Three experienced nurses in the neonatology department were trained on pain assessment among the neonates and data collection in order to compare the results immediately after invasive procedures and at intervals of 5, 10, 15 and 20 minutes. Three competently trained research assistants were responsible for enrolment of the study participants. After the evaluation of pain, the neonates were given to their mothers to breast feed to relieve pain the after the painful procedures. To assess pain, the research nurses were trained by the principal investigator (PI) on how to use the APN and NFCS scales. To increase the accuracy when using the two pain scales, a video recording was done to enable the research assistants to replay the videos and re-evaluate the neonates' responses to pain during the invasive procedures. EpiData software was used to capture data. To minimize data entry errors, two different research assistants performed double data entry. Captured data was transferred to the SPSS version 11.5 data software for analysis. The statistical tests were computed using nonparametric tests (Wilcoxon signed Ranks test and McNemar) due to skewed distribution of the data. Physiological parameters (blood pressures, heart rates and respiratory rates) were compared using Wilcoxon signed Ranks Test and this was significant if $\mathrm{P}$ value was $<0.05$.

\section{Results}

Table 1. Characteristics of the neonates $(N=60)$.

\begin{tabular}{lll}
\hline & Frequency & \% \\
\hline Gender & 31 & \\
Male & 29 & 51.7 \\
Female & 60 & 48.3 \\
Total & & 100 \\
Gestational age (weeks) & 9 & \\
$28-32$ & 16 & 15 \\
$33-36$ & 35 & 26.7 \\
$37-42$ & 60 & 58.3 \\
Total & & 100 \\
Types of procedures & 21 & \\
IV injections & 11 & 35 \\
IM injections & 28 & 18.3 \\
Phlebotomy & 60 & 46.7 \\
Total & & 100 \\
\hline
\end{tabular}

The study sample comprised $51.7 \%$ male and $48.3 \%$ female and the majority (58\%) of the neonates were born at term, at a gestational age above 37 weeks while pre-term neonates at a gestational age of 33-36 weeks were $27 \%$. Premature neonates, born at a gestational age of 28-32 weeks accounted for $15 \%$ of all the neonates. Phlebotomy was the commonest invasive procedure $(47 \%)$ and the one that contributed almost half of all the pain experienced by neonates. This was followed by $35 \%$ of the neonatal pain attributed to intravenous injections while intramuscular injections contributed $18 \%$ of the pain among the neonates (Table 1).

\section{Duration of pain after procedure}

Using appropriate techniques, reactions to pain by the neonates were monitored and documented over a 20-minute period to track the neonates' responses to pain (Table 2)

Table 2. Duration of pain $(N=60)$.

\begin{tabular}{ll}
\hline Duration (minutes) & \% Responses \\
\hline $0-5$ & 100 \\
$6-10$ & 45 \\
$11-15$ & 13 \\
$16-20$ & 6 \\
\hline
\end{tabular}

Cumulatively, all the neonates $(100 \%)$ experienced maximum pain within the immediate 5 minutes of undergoing the invasive procedures, a possible relationship with the process of piercing the skin and penetrating the deeper tissues of the neonate followed by adaptation (increasing threshold levels) to the pain through quick rise in the threshold for pain. After five minutes, there was a decline of pain among the neonates by $55 \%$, confirming further adaptation to the pain by the neonates and the short duration taken for the existence of the needles in the neonates' tissues. By the $15^{\text {th }}$ minute, $87 \%$ of the neonates could not experience pain any more. Approximately $94 \%$ of the neonates were found to have been free of pain within 16-20 minutes of the onset of the invasive procedures (Table 2).

A comparison of the duration of pain (Table 2) and the cardiorespiratory responses (Figure 1) except the heart rates show a direct relationship with the intensity of pain in which the peak responses of the respiratory rates, systolic and diastolic blood pressures with the highest (peak) responses recorded at approximately 2.5 minutes duration of the course pain. The systolic and diastolic blood pressures were analyzed using the Wilcoxon Ranks test and the changes in systolic blood pressures (SBP) immediately after inflicting pain, 5, 10, 15 and 20 minutes were found to be statistically significant at $\mathrm{p}<0.001, \mathrm{p}<0.001, \mathrm{p}<0.005$ and $\mathrm{p}<0.046$ respectively. The changes in the diastolic blood pressures (DBP) immediately after the procedures, 5 and 10 minutes, the variations were statistically significant with $\mathrm{p}<0.001$, $\mathrm{p}<0.001$ and $\mathrm{p}<0.019$ respectively. But the variation of the DBP was not significant 15 minutes and 20 minutes after the procedures with $\mathrm{p}<0.592$ and $\mathrm{p}<0.285$ respectively, implying that the effects of pain on the cardiovascular system resolved within a 15-minute period of invasive procedure. There was no observed difference between the DBP before procedure 
and 20 minutes after procedure $(p<0.582)$, meaning that the effects of pain on the diastolic blood pressure had waned within 20 minutes of venipuncture. Changes in the respiratory rates were statistically significant immediately at the commencement of the invasive procedure $(p<0.001), 5$ minutes later $(\mathrm{p}<0.001)$ and 10 minutes later $(\mathrm{p}<0.002$. The changes in the respiratory rates were not statistically significant after 15 minutes $(\mathrm{P}<0.128)$ and 20 minutes after $(p<0.74)$, implying that neonates ceased to manifest pain through physiological changes in the respiratory system after 15 minutes. With reference to the heart rates of the neonates, they significantly increased immediately after commencement of the invasive procedures $(\mathrm{p}<0.001)$ after 5 minutes $(\mathrm{p}<0.001), 10$ minutes $(\mathrm{p}<0.033)$ and after 15 minutes there was no decline in oxygen saturation $(p<0.099)$.
There was no significant change $(p<0.108) 20$ minutes after procedures, implying that neonates ceased to manifest increase in physiological changes in the heart rate after 15 minutes. The decrease in oxygen saturation immediately after procedure was significant $(\mathrm{p}<0.001)$. Oxygen saturation declined immediately at the onset of procedures and dipped significantly at 2.5 minutes, increasing significantly up to 5 minutes $(p<0.001)$ and 10 minutes $(p<0.001)$ but after 15 minutes the changes in oxygen saturation were not statistically significant $(\mathrm{p}<0.099)$ and after 20 minutes $(p<0.293)$. Thus, decline in oxygen saturation reached a peak at 2.5 minutes in response to accelerated use and persisted up to 15 minutes, thereafter, normal levels of oxygen saturation were re-established after 15 minutes.

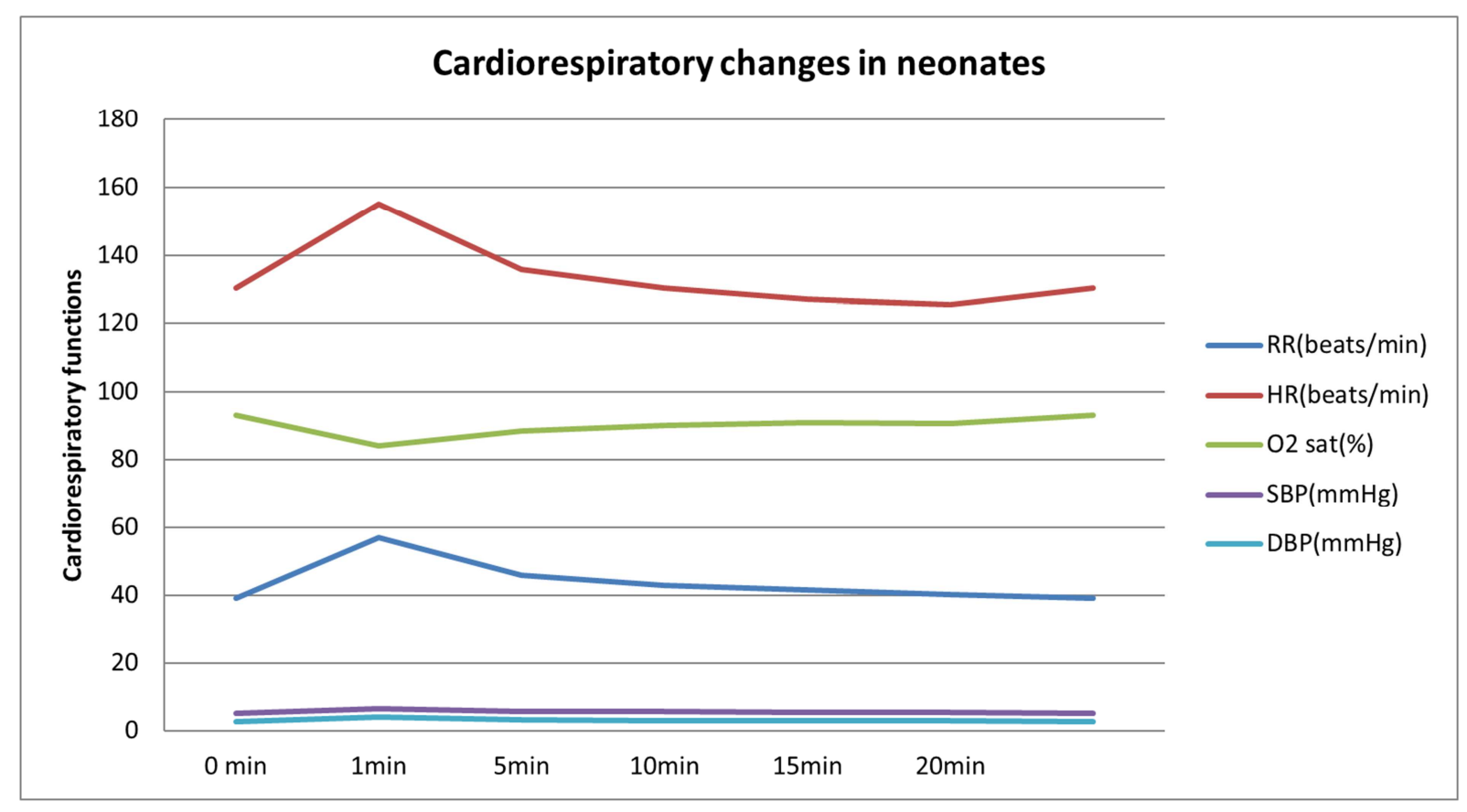

Figure 1. Cardiorespiratory changes in neonates $(N=60)$.

\section{Discussion}

The findings in this study showed that phlebotomy was the commonest invasive procedure $(47 \%)$ followed by intravenous injections (35\%) and lastly, intramuscular injections $(18 \%)$ in terms of contribution to pain among neonates. Of all the invasive procedures, $82 \%$ of the procedures were either administration of intravenous medications or venous blood sample collection for laboratory tests that were inevitable in the management of pain among the neonates. Concerning the duration of pain, all neonates $(100 \%)$ felt pain while almost half of the neonates (45\%) felt pain within 5 minutes of the procedure. At 15 minutes after procedures, only $6 \%$ of the neonates were still feeling pain and this meant that the majority of neonates $(94 \%)$ recovered from pain within 15 minutes after the invasive procedures. There had been no similar study found to compare the results within the study settings and contexts. A large majority
$(82 \%)$ of the neonates experienced very severe pain immediately the invasive procedures commenced (0-5 minutes), $15 \%$ had severe pain and 3\% had moderate pain on the NFCS scale. Carbajal et al. [16] showed that $88 \%$ of neonates felt a severe pain, a finding closely related with this study (82\%). Phlebotomy, intravenous and intramuscular injections did not differ in the degree of pain generated and this study did not find association between pain score and gestational age. While Guinsburg et al. [25] in Sao Paulo, Brazil found significant association $(\mathrm{P}<0.025)$. The difference with this study could be explained by the high accuracy of the materials used by Guinsburg. In this study, the heart rate increased immediately after invasive procedure and became normal after 15 minutes. This study differed with that of Pereira et al. [26] but they were similar in oxygen saturation levels. Respiratory rate frequency increased significantly after procedure, a finding comparable to that in India by Taksande et al [21]. Systolic and diastolic blood pressures increased during the invasive procedures and 
returned normal within 15 minutes, an exclusive finding in this study. With the results of this study, physiological parameters changed significantly in relation to invasive procedures and returned to normal 15 minutes after the procedures.

\section{Conclusion}

Invasive procedures (blood collection, intravenous administration of medications and intramuscular injections) caused acute pains among neonates at their commencement that were not catered for as part of neonatal care in Rwanda, which should be reviewed and considered during neonatal care following the findings in this study. The impact of the pain was acutely and physiologically expressed, measured and quantified justifying such measures in future and subsequent treatment and care. The huge acute cardiorespiratory neonatal responses justified training, innovation and technology as routine neonatal responses and care in Africa rather than taking for granted pain among neonates. This study, therefore, concluded that neonates recovered completely from pain inflicted by invasive procedures within 20 minutes and any effort to relieve pain should be done emphatically within the first 5 minutes of the commencement of the invasive procedures. It further concluded that acute pain is strongly present and felt by neonates yet provision has hardly been made to cater for it, leading to painful suffering of neonates in silence. The findings of this study added new information on existing pool of knowledge, thereby raising awareness further more the need for pain relief among neonates in Africa. A policy formulation is recommended to direct action.

\section{References}

[1] McGrath PJ and Unruh AM. The Social Context of Neonatal Pain. Clinical Perinatology. 2002; 29 (3): 555-72.

[2] Australian and New Zealand College of Anesthetists. Acute Pain Management: Scientific Evidence. National Health and Medical Research Council. Australian Government. Second Edition 2005.

[3] Prevention and Management of Pain in the Neonate: An Update. American Academy of Paediatrics, Committee on Fetus and Newborn and Section on Surgery, Section on Anesthesiology and Pain Medicine, Canadian Paediatric Society and Fetus and Newborn Committee. Paediatrics 2006; $118 ; 2231-41$

[4] Szymanski B. A comparative Study of Preterm Infant Pain Tools. During a Common NICU Procedure. Master's and Doctoral Projects. Paper 40. 2005 Medical college of Ohio.

[5] Cignacco E, Mueller R, Hamers J P H and Gessler P. Pain assessment in the neonate using the Bernese Pain Scale for Neonates Early Hum Dev. 2004; 78 (2): 125-31.

[6] Mathew J P and Mathew L J. Assessment and management of pain in infants. Postgraduate Medical Journal, 2003; 79: 438443.
[7] National Health and Medical Research Council. Acute Pain Management: Scientific Evidence. Commonwealth of Australia 1999. November, 1998.

[8] Stevens BJ, editor. Pain in neonates and infants: pain research and clinical management series. Elsevier; 2007.

[9] Debillon T, Zupan V, Ravault N, Magny J-F and Dehan M. Development and initial validation of the EDIN scale, a new tool for assessing prolonged pain in preterm infants. BMJ Arch Dis Child Fetal Neonatal Ed 2001; 85: F36-F41.

[10] Pitetti D R. Do No Harm but First Do Not Hurt. CMAJ • JUNE 21, 2005; 172 (13) Warnock F and Sandrin D. Comprehensive description of newborn distress behavior in response to acute pain. $2004 \mathrm{Feb} ; 07$ (3): 242-55.

[11] Gharavi, b, Schott, c, Nelle, m., Reiter, g. and Linderkamp, o. Pain management and the effect of guidelines in neonatal units in Austria, Germany and Switzerland. Pediatrics International 2007, 49: 652-658.

[12] Lago, p., Guadagni, a., Merazzi, d., Ancora, g., Bellieni, c. v., Cavazza, a. and the pain study group of the italian society of neonatology, Pain management in the neonatal intensive care unit: a national survey in Italy. Pediatric Anesthesia 2005, 15: 925-931.

[13] FitzGerald M. Developmental Biology of Inflammatory Pain. British Journal of Anesthesia 1995; 75: 177-185.

[14] Bellieni C V, Bagnoli F and Buonocore G. Alone No More: Pain in Premature Children. Ethics\& Medicine 2003, 19: 1: 510 .

[15] Riddell P R and Craig D K. Judgments of Infant Pain: The Impact of Caregiver Identity and Infant Age. J Pediatr Psychol. 2007 Jun; 32 (5): 501-11.

[16] Carbajal R, Veerapen S, Couderc S, Jugie M and Ville Y. Analgesic effect of breast feeding in term neonates: randomised controlled trial. Bmj 2003 volume 3264.

[17] MARCEAU J. Pilot study of a pain assessment tool in the Neonatal Intensive Care Unit. J. Paediatr. Child Health (2003) $39,598-601$.

[18] Gordon et al. American Pain Society Recommendations for Improving the Quality of Acute and Cancer Pain Management: American Pain Society Quality of Care Task Force. Arch Intern Med. 2005; 165 (14): 1574-1580.

[19] Anand KJ, Barton BA, McIntosh N, Lagercrantz H, Pelausa E, Young T E... and Vasa R. Analgesia and sedation in preterm neonates who require ventilatory support: results from the NOPAIN trial. Neonatal Outcome and Prolonged Analgesia in Neonates. Arch Pediatr Adolesc Med 1999 Apr: 153 (4): 331 8. Arkansas.

[20] Koot M H, Grunau E R, Tibboel D, Peters B W J, de Boer J, van Druenen J M, and Duivenvoorden J H. Neonatal Facial Coding System for assessing postoperative pain in infants: item reduction is valid and feasible. The clinical journal of pain. 2003; 19: 353-363.

[21] Taksande Amar M, Vilhekar KY, Jain M, Chitre D. Pain response of neonates to venipuncture, The Indian Journal of Paediatrics, 2005, 72 (9): 751-753.

[22] Cochran, W. G. (1977). Sampling techniques (3rd ed.). New York: John Wiley \& Sons. 
[23] Carbajal R, Paupe A, Hoenn E, Lenclen R, Olivier-Martin M. APN: evaluation behavioral scale of acute pain in newborn infants (APN). Arch Pediatr. 1997 Jul; 4 (7): 623-8.

[24] Peters JW, Koot HM, Grunau RE, de Boer J, van Druenen MJ, Tibboel D, Duivenvoorden HJ. Neonatal Facial Coding System for assessing postoperative pain in infants: item reduction is valid and feasible. Clin J Pain. 2003 Nov-Dec; 19 (6): 353-63.
[25] Guinsburg R, de Araujo PC, Branco de Almeida MF. et al. Differences in pain expression between male and female newborn infants. Pain. 2000, 85 (1-2): 127-33.

[26] Pereira, Andrea Lübe de S Thiago et al. Validity of behavioral and physiologic parameters for acute pain assessment of term newborn infants. Sao Paulo Med. J., 1999, 117 (2): 72-80. 